\title{
Beam position monitor design for a third generation light source
}

\author{
Zhichu Chen, ${ }^{*}$ Yongbin Leng, Kairong Ye, Guobi Zhao, and Renxian Yuan ${ }^{\dagger}$ \\ Shanghai Institute of Applied Physics, Chinese Academy of Sciences, Shanghai 201800, \\ China and Shanghai Synchrotron Radiation Facility, Chinese Academy of Sciences, \\ Shanghai 201204, China
}

(Received 10 June 2014; published 3 November 2014)

\begin{abstract}
The measurement of the beam orbit plays a very important role in particle accelerators. The button-type beam position monitor (BPM) was designed for the Shanghai Synchrotron Radiation Facility to reduce the impedances and to guarantee a high resolution of the measurement. Position resolution, beam impedance, higher-order mode, and impedance matching have been studied during the design based on the physical parameters of the storage ring at the Shanghai Synchrotron Radiation Facility. Meanwhile, an analytic formula of the BPM broadband impedance was derived based on a resistor-capacitor equivalent circuit. Thus, the impedance of the BPM could be analyzed quantitatively by simply measuring the capacitance of the electrode. This formula had been verified by comparing the results of the calculations of the formula and the simulations in MAFIA.
\end{abstract}

DOI: 10.1103/PhysRevSTAB.17.112801

PACS numbers: 29.20.db, 29.27.Fh, 29.85.Fj

\section{INTRODUCTION}

The beam position monitor (BPM) system at the Shanghai Synchrotron Radiation Facility (SSRF) [1,2] provides beam orbit measurements with different beam position resolution properties depending on the integration time or bandwidth setting to ensure stable and effective operations for the orbit feedback system [3], the transverse feedback system [4], the tune measurement system $[5,6]$, and the interlock system. For example, the orbit feedback system requires an information of the closed orbit of high resolution, in the range of $1 \mu \mathrm{m}$ at a rate of approximately $1 \mathrm{~ms}$, while the transverse feedback system needs bunch-by-bunch signals with a resolution in the order of $10 \mu \mathrm{m}$ at a rate in the range of $2 \mathrm{~ns}$. The tune measurement system requires turn-by-turn data with the resolution and data rate somewhere in between the previous two demands. The important technical challenges for the BPM electrodes design are beam impedance minimization, e.g., by suppression of beam excited higher-order modes (HOMs), and a good impedance matching, which will result in well-separated bunchby-bunch signals with high signal-to-noise ratio (SNR). The following sections discuss various technical details in the BPM design, as well as beam and machine conditions at the SSRF.

\footnotetext{
*chenzhichu@sinap.ac.cn

yuanrenxian@sinap.ac.cn
}

Published by the American Physical Society under the terms of the Creative Commons Attribution 3.0 License. Further distribution of this work must maintain attribution to the author $(s)$ and the published article's title, journal citation, and DOI.

\section{POSITION SENSITIVITY OF THE BPM PROBES}

The electrodes of the BPM are usually fixed in the vacuum chamber, so the geometric layout of the vacuum chamber determines the layout of the BPM, except sizes and locations of the electrodes. Figure 1 illustrates the geometric layout of the BPM electrodes in the vacuum chamber of the storage ring at the SSRF. Pairs of electrodes $(A-B$ and $C-D)$ were assembled as modules before being assembled symmetrically at the upper and lower surfaces of the vacuum chamber to complete the 4-button BPM system.

A simple relation between the output signals and the position information for a 4-button BPM is

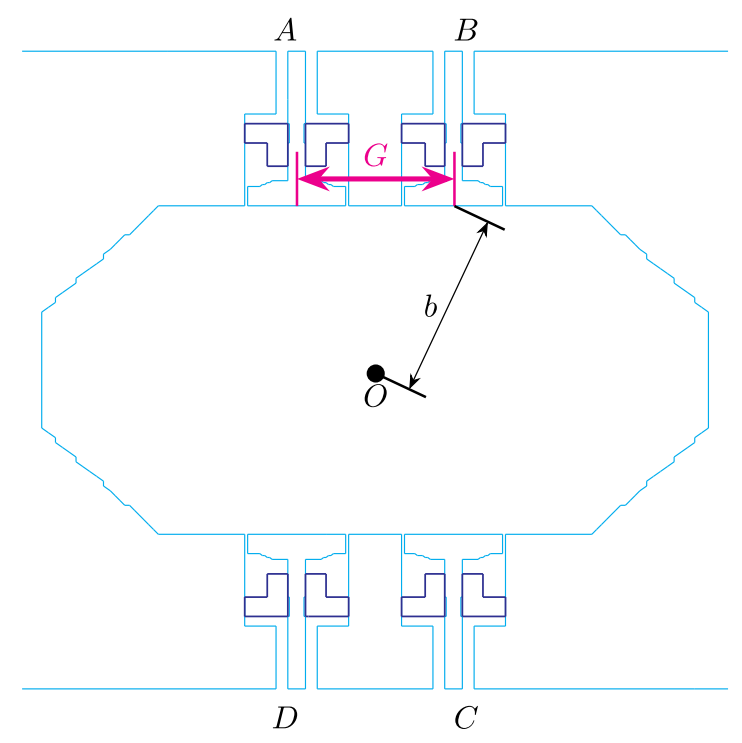

FIG. 1. Cross section layout of the SSRF 4-button BPM pickup. 
TABLE I. Simulation results of the position sensitivities of the arc BPMs and the HD BPMs at the SSRF.

\begin{tabular}{|c|c|c|c|c|c|c|c|c|}
\hline \multirow[b]{2}{*}{$\mathrm{G}(\mathrm{mm})$} & \multicolumn{4}{|c|}{ HD BPM } & \multicolumn{4}{|c|}{$\operatorname{arc~BPM}$} \\
\hline & $k_{x}\left(\mathrm{~mm}^{-1}\right)$ & $k_{y}\left(\mathrm{~mm}^{-1}\right)$ & $\rho\left(\mathrm{mm}^{-1}\right)$ & $\mathrm{g}$ & $k_{x}\left(\mathrm{~mm}^{-1}\right)$ & $k_{y}\left(\mathrm{~mm}^{-1}\right)$ & $\rho\left(\mathrm{mm}^{-1}\right)$ & $\mathrm{g}$ \\
\hline 14 & 0.0784 & 0.0913 & 0.0135 & 1.252 & 0.0504 & 0.0786 & 0.0116 & 1.340 \\
\hline 16 & 0.0863 & 0.0847 & 0.0123 & 1.179 & 0.0560 & 0.0749 & 0.0109 & 1.287 \\
\hline 18 & 0.0930 & 0.0780 & 0.0111 & 1.103 & 0.0610 & 0.0711 & 0.0102 & 1.233 \\
\hline
\end{tabular}

$$
d_{i} \approx \frac{\Delta}{k_{i} \Sigma},
$$

where $d_{i}$ is the horizontal/vertical position of the beam, $\Delta=V_{A}+V_{D}-V_{B}-V_{C}\left(\right.$ or $\left.\Delta=V_{A}+V_{B}-V_{C}-V_{D}\right)$ is the difference voltage, sensitive to the horizontal (vertical) beam displacement, $\Sigma=V_{A}+V_{B}+V_{C}+V_{D}$ is the sum signal of the electrodes, $V_{\alpha}$ 's are the output voltage signals from the 4 electrodes and $k_{i}$ is a linear calibration constant. For small beam displacements from the center of the beam pipe, the resolution of the BPM can be expressed as: [[7], Eq. (9.1)][[8], pp. 54]

$$
\delta d_{i}=\frac{1}{2 k_{i} \cdot S N R},
$$

where $S N R$ is the signal-to-noise ratio of the BPM electrodes with the attached read-out electronics channel. From Eq. (2) it follows that the BPM resolution improves with higher beam intensities, $V_{\alpha}$ 's increase, as well as with a BPM electrode configuration that would give a higher normalized position sensitivity $\delta d_{i}$.

Two types of BPMs are installed at the SSRF arc areas and drift lines, called the arc BPMs and the high-definition (HD) BPMs, respectively. The dimensions of the octagonal vacuum chambers are $68 \mathrm{~mm} \times 34 \mathrm{~mm}$ and $100 \mathrm{~mm} \times$ $26 \mathrm{~mm}$ for arc and HD BPMs, respectively. The closed orbit data of the HD BPMs are used for the orbit feedback system, therefore a high sensitivity was a more important design parameter for the HD BPMs than for the arc BPMs. The mechanical module that contains two electrodes was designed and standardized to reduce the cost and simplify the installation, while keeping alignment errors still under control. The cross-section geometry of the HD BPM was designed such that horizontal and vertical sensitivities are similar, however, using the standardized BPM pair in the arc BPM housing results in different sensitivity properties.

The sensitivity of the BPM pickup could be estimated analytically, e.g. based on the mirror charge model. For the given octagon shape of the BPM cross-section, the numerical solution of the 2D Laplace problem is valid, as of the relativistic properties of the high-energy electron beam, and promises a more accurate result [9]. The output signal of the electrode was obtained by evaluating the distribution of the induced charge on the inner surface of the vacuum chamber, from which the sensitivity was computed.
Some results of the simulations are listed in Table I, where $G$ is the distance between the adjacent electrodes (as shown in Fig. 1), $k_{x, y}$ the horizontal/vertical calibration factor, which is equivalent to the BPM sensitivity, $\rho$ the normalized, mirror charge density of the beam induced charge [see the definition in Eq. (3)] and $g$ the shape factor. $\rho$ is determined by the structure of the vacuum chamber and the location and shape of the BPM electrode. $g=\rho \times 2 \pi b$ is a geometrical form factor, expressing the difference of the induced charge of the actual BPM cross-section to a cylindrical cross-section of radius $b$ (as shown in Fig. 1). $G$ was chosen to be $16 \mathrm{~mm}$ at the SSRF.

\section{TRANSFER IMPEDANCE AND OUTPUT SIGNALS OF THE BPM}

While the position sensitivity has some effect on the BPM resolution, the output signal level at the BPM electronics directly influence the SNR, and is therefore more important for the resolution. Most of the noise contribution is related to the thermal noise at the load impedance, which is further amplified by the gain stages of the front-end electronics, signal processor and other subsequent processes.

The diameter of the button electrode has strong influence on the transfer impedance, thus on the output signal and the coupling impedance. It has to be optimized to give sufficient output signal levels under various beam conditions, i.e., the beam current at the SSRF ranges $0.2 \mathrm{~mA}-$ $400 \mathrm{~mA}$, to ensure a reliable operation of the BPM system.

Since the velocity of the high energy electrons is quite close to the light speed, the related electromagnetic field is focused in the transverse plane, based on the theory of special relativity. The induced charge on the electrode is proportional to the beam current due to Gauss's law. Consider a rectangular button with an infinitesimal width $\mathrm{d} x$ (as shown in Fig. 2), the induced charge on the button would be

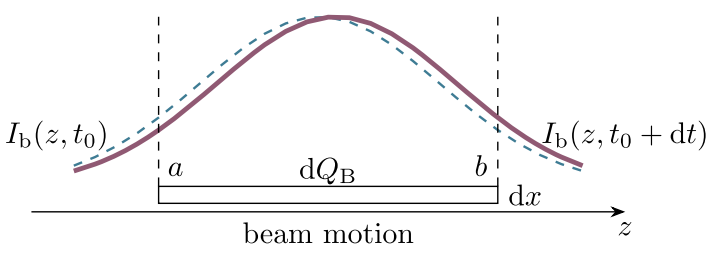

FIG. 2. Induced charge and current on a line button. 


$$
\mathrm{d} Q_{\mathrm{B}}(t)=\rho \mathrm{d} x \int_{a}^{b} \frac{I_{\mathrm{b}}(z, t)}{c_{0}} \mathrm{~d} z
$$

where $\mathrm{d} Q_{\mathrm{B}}$ is the induced charge on the button, $\rho$ is the linear charge density, $I_{\mathrm{b}}(z, t)=\frac{Q_{0}}{\sqrt{2 \pi} \sigma_{t}} \exp \left[-\frac{\left(t-z / c_{0}\right)^{2}}{2 \sigma_{t}^{2}}\right]$ is the longitudinal current distribution of the Gaussian bunch and $c_{0}$ is the speed of light. The induced current is the rate of change of the charge:

$$
\mathrm{d} I_{\mathrm{B}}(t)=\rho\left[I_{\mathrm{b}}(b, t)-I_{\mathrm{b}}(a, t)\right] \mathrm{d} x .
$$

This means that the induced current on the button is proportional to the change of the current of the beam within the button area.

For a small circular button, the linear charge density of the induced charge is approximately linear to the $x$-coordinate: $\rho(x) \simeq \rho+\alpha x$. The induced current on the whole button could be obtained by integrating over the $x$-axis (as shown in Fig. 3):

$$
I_{\mathrm{B}}(t)=\int_{-r}^{r} \rho(x)\left\{I_{\mathrm{b}}(b(x), t)-I_{\mathrm{b}}[a(x), t]\right\} \mathrm{d} x .
$$

For a circular electrode (as shown in Fig. 3), the induced current is

$$
\begin{aligned}
I_{\mathrm{B}}(t)= & \int_{0}^{\pi}(\rho+\alpha r \cos \theta) \\
& \times\left[I_{\mathrm{b}}(r \sin \theta, t)-I_{\mathrm{b}}(-r \sin \theta, t)\right] r \sin \theta \mathrm{d} \theta .
\end{aligned}
$$

Thus the induced current in the frequency domain can be written as

$$
\begin{aligned}
I_{\mathrm{B}}(\omega) & =I_{\mathrm{b}}(\omega) \int_{-\pi}^{0}(\alpha r \cos \theta+\rho)\left[1-\exp \left(\frac{2 j \omega r \sin \theta}{c_{0}}\right)\right] r \sin \theta \mathrm{d} \theta \\
& =I_{\mathrm{b}}(\omega) \rho r\left[-2-\int_{-\pi}^{0} \sin \theta \exp \left(\frac{2 j \omega r \sin \theta}{c_{0}}\right) \mathrm{d} \theta\right] \\
& =I_{\mathrm{b}}(\omega) \rho r\left[-2-j \pi J_{1}\left(\frac{2 \omega r}{c_{0}}\right)+\pi H_{-1}\left(\frac{2 \omega r}{c_{0}}\right)\right], \quad \text { for } \frac{2 \omega r}{c_{0}} \in \mathbb{R},
\end{aligned}
$$

where $r$ is the radius of the electrode. $J_{1}$ and $H_{-1}$ denote the Bessel function of the first kind and the Struve function, respectively, where $J_{\nu}(z)$ satisfies

$$
z^{2} y^{\prime \prime}+z y^{\prime}+\left(z^{2}-\nu^{2}\right) y=0,
$$

and $H_{\nu}(z)$ satisfies

$$
z^{2} y^{\prime \prime}+z y^{\prime}+\left(z^{2}-\nu^{2}\right) y=\frac{2}{\pi} \frac{z^{\nu+1}}{(2 \nu-1) ! !} .
$$

To simplify the calculation, the electrode is regarded as a rectangle whose sides are $2 r / \kappa$ and $\kappa \pi r / 2$, where $\kappa$ is a constant and is chosen to be 1 in this case, when the bunch length is larger than or approximately equal to the radius of the electrode. In that case, the induced current can be written as:

$$
I_{\mathrm{B}}(\omega) \simeq \rho \frac{\kappa \pi r}{2}\left[1-\exp \left(\frac{2 j \omega r}{\kappa c_{0}}\right)\right] I_{\mathrm{b}}(\omega) .
$$

The induced current of the BPM electrode $I_{\mathrm{B}}(\omega)$ will be released through the $50 \Omega$ load and the capacitor of the electrode, as shown in the equivalent circuit in Fig. 4 inspired by [10]. The input voltage of the BPM electrode thus could be written as:

$$
U_{\mathrm{B}}(\omega)=I_{\mathrm{B}}(\omega) \frac{R+j \omega L}{1-\omega^{2} L C+j \omega C R}
$$

where $R=50 \Omega$ is the load and $C$ the capacitance between the electrode and the ground which is in the range of $1 \mathrm{pF}$ and $10 \mathrm{pF}$ depending on the size and geometry of the button in most cases. The capacitive reactance is far greater than the resistance of the load at the working frequency, $500 \mathrm{MHz}$ at the SSRF, of the electronics of the BPM, so the output voltage is primarily contributed by the resistor

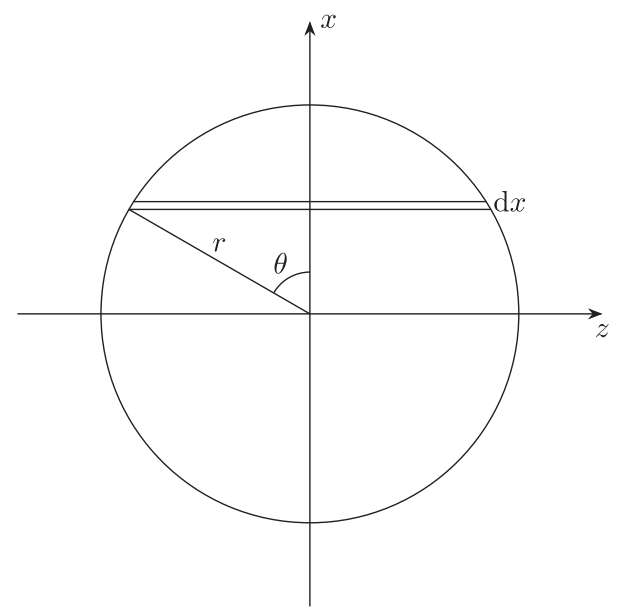

FIG. 3. Induced current on a circular button. 


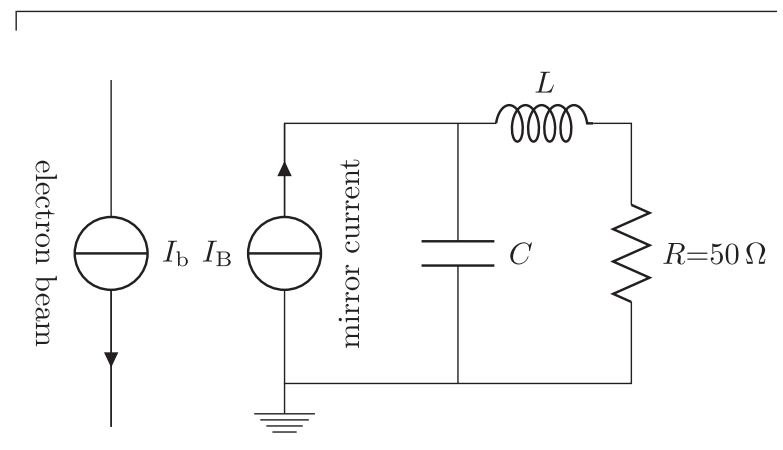

FIG. 4. Equivalent circuit.

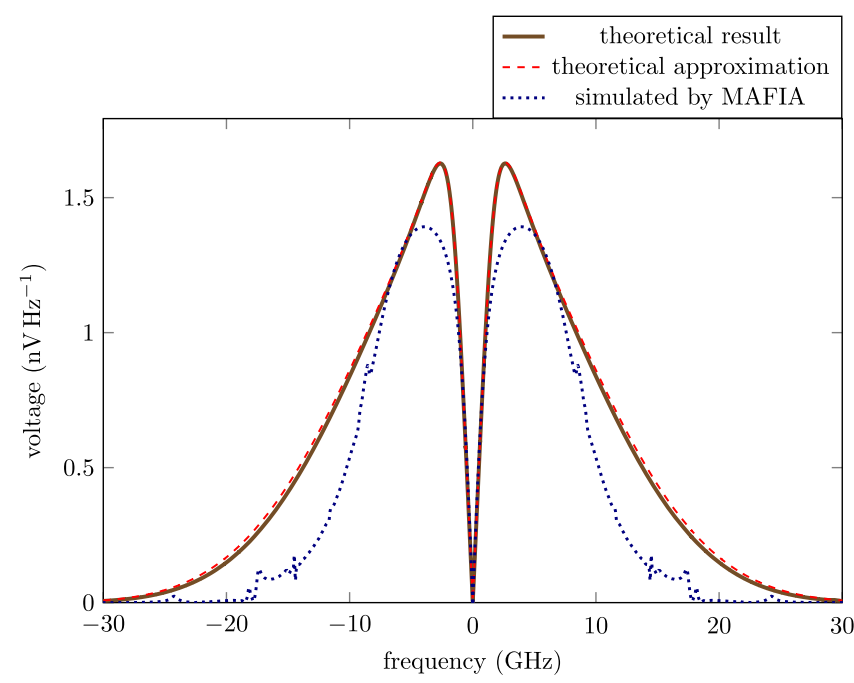

FIG. 5. Output spectra of the BPM electrode simulated by MAFIA and calculated with Eq. (8).

$R$. Therefore, the capacitance is negligible when estimating resolution of the BPM electrodes.

The calculated spectrum [using Eq. (8) and assuming $L=2.3 \mathrm{nH}$ and $C=2.2 \mathrm{pF}]$ of the output signal of the BPM electrode and the simulated one (using MAFIA) are plotted in Fig. 5. The power and the SNR of the signals at different beam currents to be input to the BPM electronics are listed in Table II.

TABLE II. The power and the SNR of the signals at different beam currents to be input to the BPM electronics $(10 \mathrm{MHz}$ bandwidth).

\begin{tabular}{lcc}
\hline \hline Beam current $(\mathrm{mA})$ & $P(d B m)$ & SNR $(\mathrm{dB})$ \\
\hline 0.2 & -64.4 & 66.4 \\
0.7 & -54.0 & 76.8 \\
1 & -50.7 & 80.1 \\
10 & -30.7 & 100.1 \\
100 & -10.7 & 120.1 \\
300 & -1.2 & 129.6 \\
400 & 1.3 & 132.1 \\
\hline \hline
\end{tabular}

As noticed, the magnitude of the simulation result does not agree with the theoretical one thoroughly. The lumpedparametric model does not qualify on all frequencies, but the results are close enough in practice.

\section{WAKEFIELD IMPEDANCE}

The output signal of the BPM electrode should be large, to improve the resolution. However, to achieve large signal levels requires physical large area electrodes and wide gap dimensions, which can lead to substantial wakefield effects, and contribute to beam instabilities, as well as to local RF heating effects in the BPM electrodes.

The beam coupling, or wakefield impedance has been studied in detail, so that the dimensions of the BPM electrode can be optimized during the design stage. Based on impedance formulas [11-13] and inspired by the lumped model method [10], a new model was established which not only can determine the dimensions of the electrode-such as the gap between the button and the vacuum chamber, the diameter and the thickness of the button-during the design stage, but also provide an approach to estimate its coupling impedance.

A wakefield is generated when the beam passes the electrodes. The real (resistive) part of the equivalent longitudinal wake-impedance corresponds to the energy loss of the beam, and the imaginary (reactive) part is related to the energy stored in the capacitance of the button electrode, i.e., the virtual work. In case of short range wake fields caused by a broadband button electrode, the imaginary part of the wake-impedance is responsible for the energy exchange between head and tail of the bunch. Even though this effect does not cause power losses to the beam bunch, it drives a longitudinal oscillation between bunch head and tail, thus may cause head-tail instabilities of a bunch or multibunch coupling instabilities under certain circumstances.

From Eqs. (7) and (8), the consumed power spectrum density $[7,10]$ of the induced current can be written as the follows, considering only transverse field components of the beam passing the electrode:

$$
\begin{aligned}
P_{\mathrm{B}}(\omega)= & I_{\mathrm{B}}^{*}(\omega) U_{\mathrm{B}}(\omega) \\
= & \left|I_{\mathrm{b}}(\omega)\right|^{2} 2 \kappa^{2} \rho^{2} r^{2} \\
& \times\left[1-\cos \left(\frac{\omega}{\omega_{\mathrm{B}}}\right)\right] \frac{R+j \omega\left(L-\omega^{2} L^{2} C-R^{2} C\right)}{\left(1-\omega^{2} L C\right)^{2}+(\omega C R)^{2}},
\end{aligned}
$$

where $\omega_{\mathrm{B}}=c_{0} / 2 \kappa r$ is the characteristic frequency of the electrode. The real part of the right-hand side of Eq. (9) expresses the energy loss of the induced current on the load and the imaginary part is the capacity of the energy stored in the capacitance of the button electrode. The energy losses of the beam due to the wakefield is the real part of the following coupling power spectrum density: 


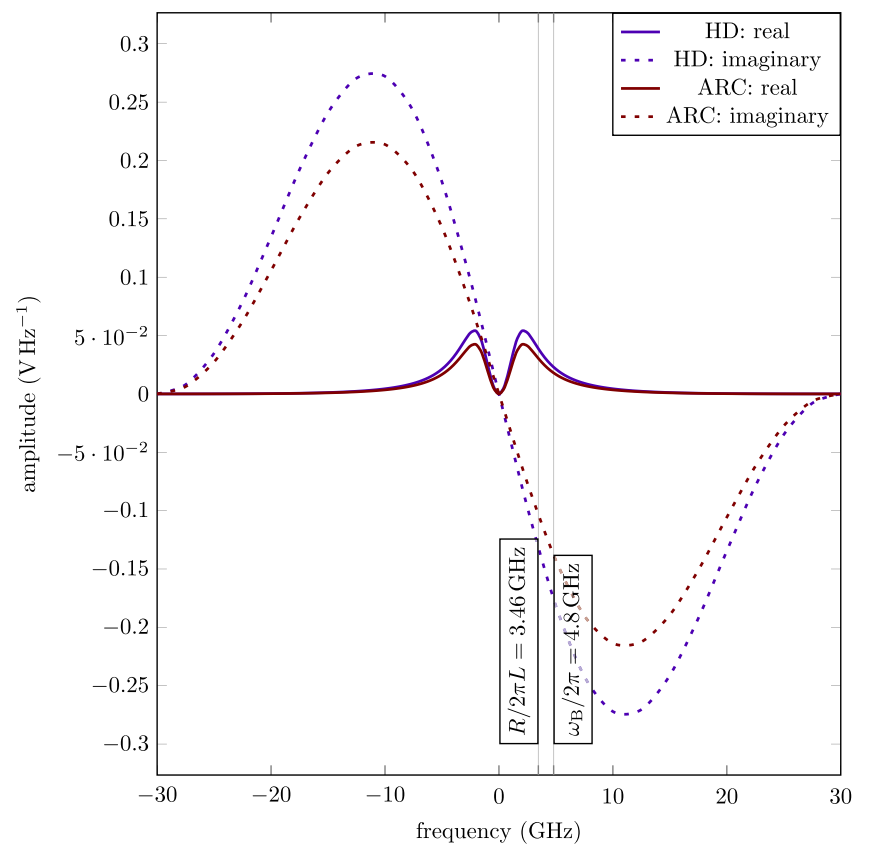

FIG. 6. Wakefield impedance spectra of a single button.

$$
P_{\mathrm{c}}(\omega)=I_{\mathrm{b}}^{*}(\omega) \cdot\left[I_{\mathrm{b}}(\omega) Z(\omega)\right]=\left|I_{\mathrm{b}}(\omega)\right|^{2} Z(\omega),
$$

where $Z(\omega)$ is the wakefield impedance of the BPM. Since only the real part of $Z(\omega)$, contributes for energy consumption, the energy loss of the beam has to be equal to the work on the load resistance. Define $Z_{R}(\omega)$ as the longitudinal wakefield impedance which comes from the load. Comparing the real parts of Eqs. (9) and (10), the real part of $Z_{R}(\omega)$ is expressed as:

$$
\Re\left[Z_{R}(\omega)\right]=\frac{2 \kappa^{2} \rho^{2} r^{2}\left[1-\cos \left(\omega / \omega_{\mathrm{B}}\right)\right]}{\left(1-\omega^{2} L C\right)^{2}+(\omega C R)^{2}} R
$$

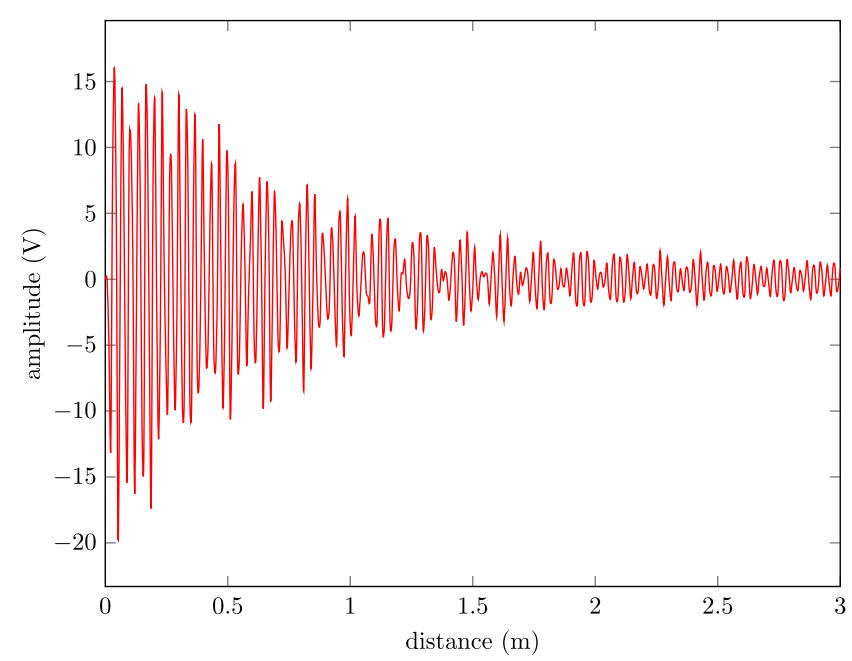

FIG. 7. The longitudinal wakefield potential of the BPM simulated by MAFIA.

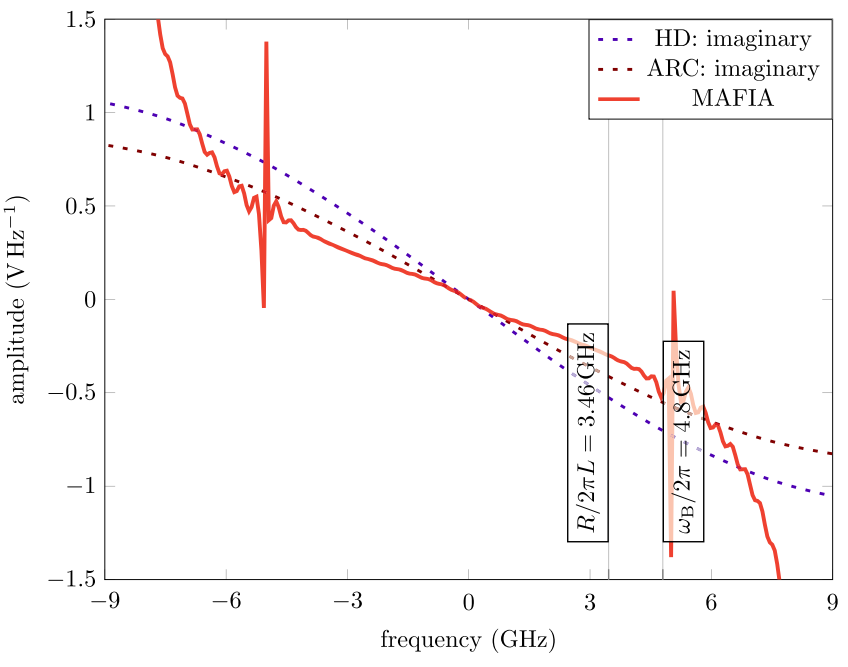

FIG. 8. Spectrum of the longitudinal wakefield potential of the HD-BPM simulated by MAFIA. The analytically calculated ones are listed for comparison. The results shown in Fig. 6 have been multiplied by 4 since there are four buttons in a BPM.

The longitudinal wake-impedance is the Fourier transformation of the time domain wakefield. The real and the imaginary part of the analytic function $Z_{R}(\omega)$ satisfies the following Hilbert transform relation: $[10,14]$

$$
\Im\left[Z_{R}(\omega)\right]=-\frac{1}{\pi} \text { p.v. } \int_{-\infty}^{\infty} \frac{\Re\left[Z_{R}\left(\omega^{\prime}\right)\right]}{\omega-\omega^{\prime}} \mathrm{d} \omega^{\prime},
$$

where p.v. stands for the Cauchy principal value.

Considering the fact, that the effective load impedance for the induced current on the button electrode is bandwidth limited, the limits of the integral in Eq. (12) can be reduced to a range $[-R / L, R / L]$, which results to the imaginary part of the wakefield impedance due to the load:

$$
\Im\left[Z_{R}(\omega)\right]=-\frac{\kappa^{2} \rho^{2} r^{2} \sin (\omega R C)}{\omega_{\mathrm{B}}^{2} R^{2} C^{2}\left[1+(\omega R C)^{2}\right]} R .
$$

As the capacitance of the load circuit does not consume energy, the real part of that contribution to the wakeimpedance equals to zero. By comparing the imaginary parts of Eqs. (9) and (10), the imaginary part of this part of the impedance can be written:

$$
Z_{C}(\omega)=2 \kappa^{2} \rho^{2} r^{2}\left[1-\cos \left(\frac{\omega}{\omega_{\mathrm{B}}}\right)\right] \frac{\omega\left(L-\omega^{2} L^{2} C-R^{2} C\right)}{\left(1-\omega^{2} L C\right)^{2}+(\omega C R)^{2}} .
$$

Thus the total impedance $Z(\omega)$ is obtained: $Z(\omega)=$ $Z_{R}(\omega)+j Z_{C}(\omega)$.

From these results it turns out, for bunch lengths substantially larger than the diameter of the electrode, i.e., the beam spectrum is well below the characteristic frequency of the electrode, the imaginary part of the 
TABLE III. The results of the wakefield impedances when varying the button thickness.

\begin{tabular}{llllll}
\hline \hline Parameter & \multicolumn{5}{c}{ Button thickness (mm) } \\
\cline { 2 - 6 } & \multicolumn{1}{c}{1} & \multicolumn{1}{c}{2} & \multicolumn{1}{c}{3} & \multicolumn{1}{c}{5} \\
\hline adjusted capacitance (pF) & 1.56 & 2.20 & 3.09 & 3.85 & 4.61 \\
impedance@1.5 GHz (MAFIA) & 0.35 & 0.234 & 0.18 & 0.165 & 0.143 \\
effective inductance (nH) (MAFIA) & 0.0262 & 0.0183 & 0.0148 & 0.0124 & 0.0101 \\
impedance@1.5GHz (model) & 0.349 & 0.236 & 0.178 & 0.143 & 0.12 \\
effective inductance (nH) (model) & 0.0248 & 0.0168 & 0.0127 & 0.0102 & 0.0085 \\
\hline \hline
\end{tabular}

wake-impedance contribution is mainly caused by the resistive load impedance [11,12], see Eq. (13). On the other hand, for bunch lengths substantially shorter than the diameter of the electrode, the wake-impedance contribution is mainly caused by the capacitance of the equivalent load circuit, given in Eq. (14). In third generation light sources, such as the SSRF, the bunch length is typically of similar dimension as the electrodes of the BPM pickups. The beam spectrum often extends to frequencies of $10 \mathrm{GHz}$, or beyond. $Z_{C}(\omega)<\Im\left[Z_{R}(\omega)\right]$ at low frequencies $\omega<R / L$ and $Z_{C}(\omega)>\Im\left[Z_{R}(\omega)\right]$ at high frequencies. For our typical bunch length ( $4.2 \mathrm{~mm}$ or $14 \mathrm{ps})$ at the SSRF the wakeimpedance has to be determined by the external load as well as the capacitance of the electrode, i.e., Eqs. (11), (13), and (14), and none of them should be ignored.

Based on the above results, related calculations and simulations have been performed at the SSRF. After some simple computations, the capacitance of the button electrode was estimated to be $1.9 \mathrm{pF}$. The capacitance and the inductance of the ceramic section, required to seal the vacuum, was estimated with $0.5 \mathrm{pF}$ and $2.3 \mathrm{nH}$, respectively. So the values of the equivalent circuit total to $2.4 \mathrm{pF}$ and $2.3 \mathrm{nH}$, respectively. The capacitance was later adjusted to $2.2 \mathrm{pF}$ based on our measurements.

Figure 6 shows, at low frequencies, i.e., $\omega<R / L$, the wake-impedance is dominated by the external load. At $|\omega| \ll \omega_{\mathrm{B}}$, the imaginary part is proportional to the frequency. At those low frequencies, the broadband impedance $|Z(\omega) / n|\left(n=\omega / \omega_{\text {rev }}\right.$ is the harmonic number) of a single HD-BPM electrode computes to $27.3 \mu \Omega$, so the total impedance for the BPM with four electrodes is $109.2 \mu \Omega$, and the equivalent inductance is $0.0250 \mathrm{nH}$. As at higher frequencies, the wake-impedance does not follow this linear factor with frequency, the effective longitudinal coupling impedance [[15], Eq. (5)][[16], Eq. (2.1)] was modified to match the computations, using an equivalent inductance of $0.0168 \mathrm{nH}$.

Figure 7 shows the time domain longitudinal wake potential of a MAFIA simulation of our BPM. The real and the imaginary parts of the equivalent wake impedance are derived simply by a Fourier transformation [17]. The imaginary part of the spectrum in Fig. 8 was obtained directly from the wake potential, which fits to an effective longitudinal coupling impedance of $102.2 \mu \Omega, 0.0183 \mathrm{nH}$.
The simulated and calculated spectra show a reasonable agreement for $|\omega|<7 \mathrm{GHz}$ ignoring the HOM effects.

As the height of the arc BPM vacuum chamber is larger than that of the HD-BPM, the comparison of both cases for the longitudinal wake-impedances are shown in Fig. 6. For the arc BPM the broadband impedance at low frequencies and the effective longitudinal coupling impedance, based on our model, are found to be $0.0196 \mathrm{nH}$ and $0.0132 \mathrm{nH}$, respectively, while the MAFIA simulation returns $0.0137 \mathrm{nH}$ as effective longitudinal coupling impedance.

Further studies of the wake-impedance for button electrodes of different thickness are summarized in Table III, using both the analytical model and the MAFIA simulation. The simulation results confirm, thinner buttons tend to have a higher wake-impedance.

\section{HIGHER ORDER MODE RESONANCES}

The bunch length at the SSRF is $4.2 \mathrm{~mm}$ and the related frequency spectrum extents to $10 \mathrm{GHz}$ and above. Beside the TEM signal transmission and the broadband wake potential, also narrow HOM resonances of the button electrode can be excited by the passing bunches, in particular the $\mathrm{TE}_{11}$ mode, which determines frequency and amplitude of the dominant narrow-band impedance, and was studied in theory [18-20] and by numerical simulations $[21,22]$. The BPM at the SSRF was simulated with MAFIA and two narrow-band resonant peaks could be observed, near $9 \mathrm{GHz}$ and $10.7 \mathrm{GHz}$, with $25 \Omega$ and $14 \Omega$

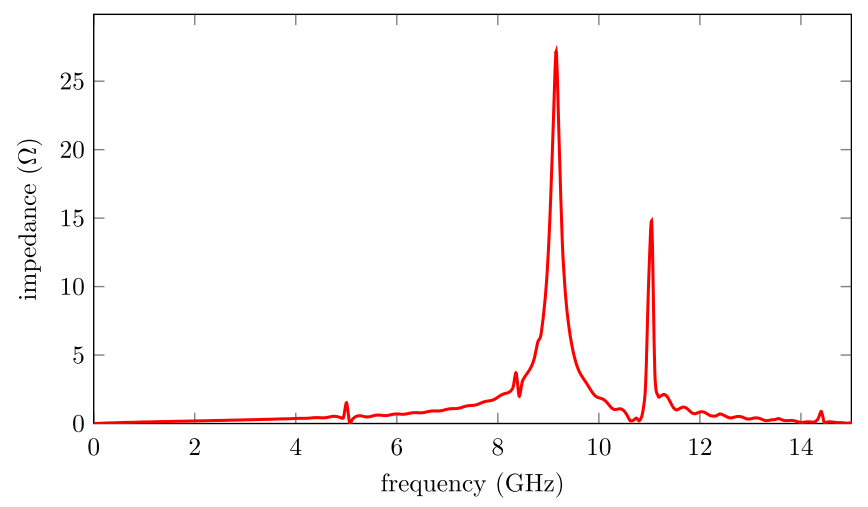

FIG. 9. Wakefield impedance of the BPM simulated in MAFIA shows the HOMs' influences. 
TABLE IV. Calculated characteristic frequencies inside the electrode (denoted as $f_{\text {electrode }}$ ) and cutoff frequencies of the vacuum chamber (denoted as $f_{\text {cutoff }}$ ) of the HOMs.

\begin{tabular}{lcc}
\hline \hline Mode number & $f_{\text {electrode }}(\mathrm{GHz})$ & $f_{\text {cutoff }}(\mathrm{GHz})$ \\
\hline 1 & 5.4 & 2.449 \\
2 & 9.1 & 4.412 \\
3 & 10.7 & 4.732 \\
4 & 12.15 & 4.800 \\
5 & 12.24 & 5.464 \\
6 & 14.48 & 6.322 \\
7 & 14.52 & 6.729 \\
8 & 15.23 & 7.220 \\
9 & 15.31 & 8.201 \\
10 & 17.29 & 8.259 \\
\hline \hline
\end{tabular}

impedance, respectively, see Fig. 9. The first resonance is related to the $\mathrm{TE}_{11}$ eigenmode of the electrode, the second is a higher order eigenmode, also listed in Table IV. One of the important BPM design criteria was to avoid any eigenmode excitation of the BPM pickup by the SSRF $500 \mathrm{MHz}$ power RF fundamental frequency, and any of those higher order harmonics.

The TE modes trapped in the vacuum chamber will be coupled in the output signal of the electrode if eigenfrequencies are in the range of the working frequencies of the electronics [23,24], so the corresponding errors in position measurements should be considered. The cutoff frequency of the $\mathrm{TE}_{01}$ mode is substantially higher than the $500 \mathrm{MHz}$ operation frequency of the signal processing electronics (see Fig. 9 and Table IV). The TE modes will not contribute significantly in position measurements.

\section{IMPEDANCE MATCHING AND ELECTRODE CAPACITANCE}

The induced current passes through a ceramic seal before it is extracted to the electronics (as shown in Fig. 10). This ceramic structure is used to seal the vacuum and impedance matching is required to avoid internal resonances. The impedance matching can be performed in two ways: changing the structure of the conductor $[21,22]$ or changing the permittivities of the insulating medium [18]. The diameter of the neck of the feedthrough, where the ceramic seal is allowed to be expanded, was optimized by changing it from $0.92 \mathrm{~mm}$ to $1.5 \mathrm{~mm}$. In the original design, the neck of the ceramic seal had a deviation of $\pm 10 \Omega$ from the nominal $50 \Omega$ characteristic impedance. The step response measured with a time-domain reflectometer (TDR) shows how the reflections could be reduced by modifying the dimensions of that neck (see Fig. 11).

Since the wavelengths of the frequencies of the beam signals are still large compared to the BPM electrode dimensions, the signal transmission is based on transverse electromagnetic (TEM) modes, allowing a straightforward calculation of the characteristic impedance.

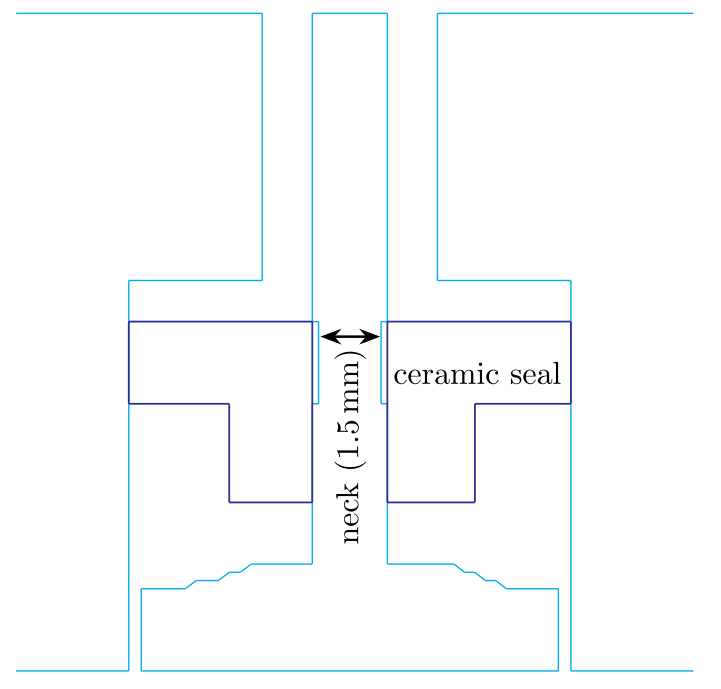

FIG. 10. The feedthrough structure of the SSRF BPM.

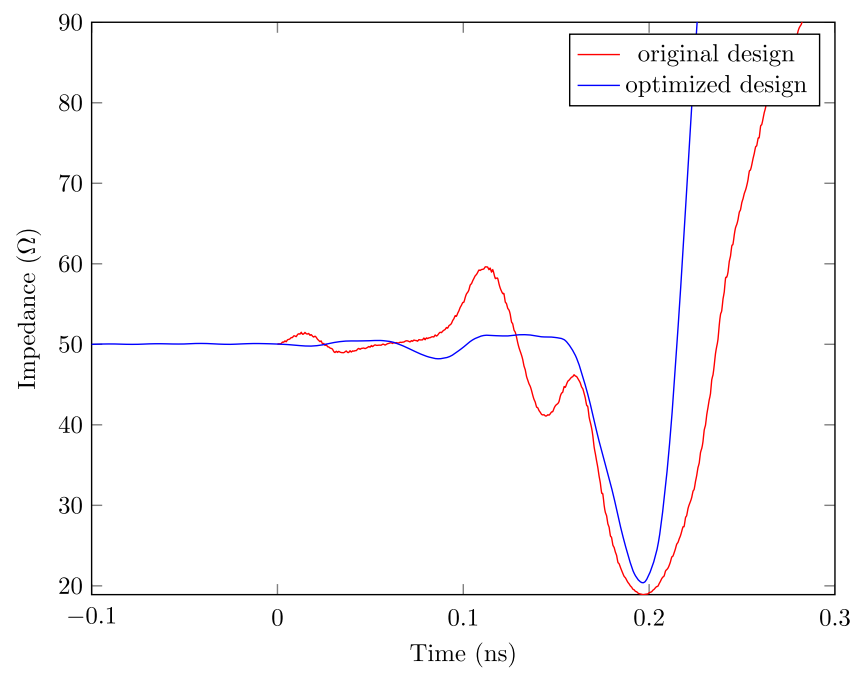

FIG. 11. Step response of the electrode measured at the SSRF. The radius of the neck in the original design is $0.92 \mathrm{~mm}$ and it's optimized to be $1.5 \mathrm{~mm}$.

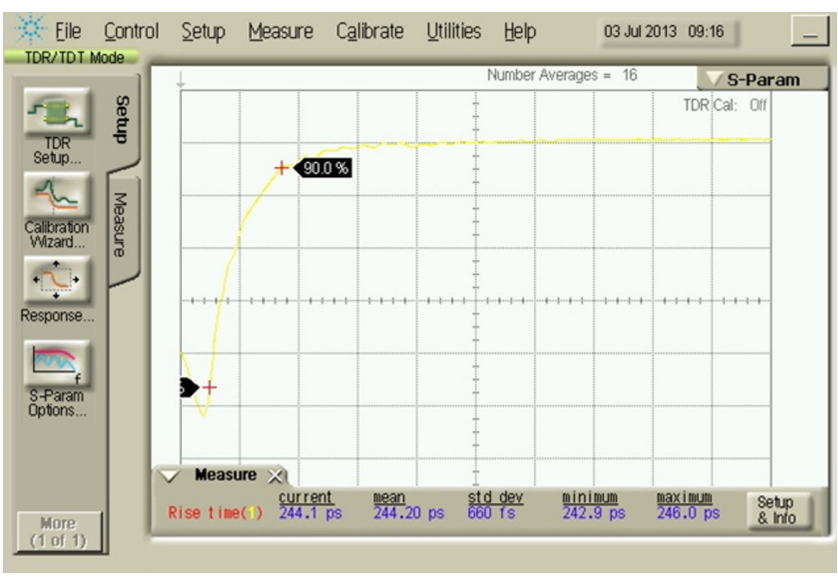

FIG. 12. The rise time of the open-circuit BPM. 
The shunt capacitance electrode-to-ground is $954 \mathrm{pFm}^{-1}$. The effective permittivity of the upper part of the ceramic seal is 5.3 so the corresponding characteristic impedance is $50.2 \Omega$ and the shunt capacitance is $150 \mathrm{pFm}^{1}$. The effective permittivity of the lower part of the ceramic seal is 2.55 so the corresponding characteristic impedance is $66 \Omega$ and the shunt capacitance is $80 \mathrm{pFm}^{1}$. The total capacitance is $2.4 \mathrm{pF}$ and is confirmed by measuring the rise time with a TDR. The result of the open-circuit probe is 244 ps (from $10 \%$ to $90 \%$, as shown in Fig. 12), i.e., the equivalent capacitance is $2.2 \mathrm{pF}$, which is expected from the model.

\section{CONCLUSIONS}

The estimate of the impedance of the BPM electrode is essential in a modern accelerator. This article studies the feasibility of using a lumped-parameter model, with measurable circuit elements, in place of computer simulations in the design stage. The analytical approach will be able to fine-tune BPM design parameters quickly without having to iterate a time-consuming numerical calculation over parameter values.

The button-style BPM of the SSRF was analyzed in terms of transfer impedance and sensitivity, and with some additional focus on the wake-impedance. The wakefield results, based on the analytical model, derived from a simple equivalent circuit, showed a good agreement with the numerical simulations.

[1] Z. Zhao and H. Xu, in Proceedings of the 9th European Particle Accelerator Conference, Lucerne, 2004 (EPS-AG, Lucerne, 2004) [http://accelconf.web.cern.ch/AccelConf/ e04/].

[2] Z. Zhao, H. Xu, and H. Ding, in Proceedings of the 21st Particle Accelerator Conference, Knoxville, TN, 2005 (IEEE, Piscataway, NJ, 2005), p. 214.

[3] J. Hou, S. Tian, M. Zhang, Y. Xu, and G. Liu, Chin. Phys. C 33, 145 (2009).

[4] R. Yuan, Y. Leng, L. Yu, L. Han, and K. Ye, Science China, Physics, Mechanics \& Astronomy 54, S305 (2011).

[5] Y. Leng, W. Zhou, Y. Chen, K. Ye, R. Yuan, Y. Yan, Y. Zou, G. Huang, G. Zhao, Z. Chen, L. Yu, J. Chen, J. Yu, C. Yin, L. Zhao, and D. Liu, in Proceedings of the 11th European
Particle Accelerator Conference, Genoa, 2008 (EPS-AG, Genoa, Italy, 2008), p. 1080.

[6] G. Roberto Aiello, R. G. Johnson, D. J. Martin, M. R. Mills, J. J. Olsen, and S. R. Smith, in AIP Conf. Proc. 390, 341 (1997).

[7] R. E. Shafer, IEEE Trans. Nucl. Sci. 32, 1933 (1985).

[8] S. R. Smith, in AIP Conf. Proc. 390, 50 (1997).

[9] T. Shintake, M. Tejima, H. Ishii, and J. ichi Kishiro, Nucl. Instrum. Methods Phys. Res., Sect. A 254, 146 (1987).

[10] F. Marcellini, M. Serio, A. Stella, and M. Zobov, Nucl. Instrum. Methods Phys. Res., Sect. A 402, 27 (1998).

[11] S. S. Kurennoy and G. V. Stupakov, Part. Accel. 45, 95 (1994).

[12] S. S. Kurennoy, R. L. Gluckstern, and G. V. Stupakov, Phys. Rev. E 52, 4354 (1995).

[13] S. S. Kurennoy, IEEE Trans. Microwave Theory Tech. 44, 1109 (1996).

[14] Morse and Feshbach, Methods of Theoretical Physics (McGraw Hill, New York, 1953) p. 373.

[15] F. J. Sacherer, IEEE Trans. Nucl. Sci. 24, 1393 (1977).

[16] B. Zotter, Report No. CERN/ISR-TH/80-03, 1980.

[17] R. Klatt, F. Krawczyk, W.-R. Novender, C. Palm, T. Weiland, B. Steffen, T. Barts, M. J. Browman, R. Cooper, C. T. Mottershead, G. Rodenz, and S. G. Wipf, in Proceedings of the 1986 Linear Accelerator Conference (SLAC, Stanford, CA, 1986), p. 276 [SLAC-Report-303].

[18] T. Obina, T. Shintake, Y. H. Chin, and N. Akasaka, in Proceedings of the Particle Accelerator Conference, Dallas, TX, 1995 (IEEE, New York, 1995).

[19] G. V. Stupakov and S. S. Kurennoy, Phys. Rev. E 49, 794 (1994).

[20] F. Caspers and T. Scholz, Part. Accel. 51, 251 (1996).

[21] C. K. Ng, T. Weiland, D. Martin, S. Smith, and N. Kurita, in Proceedings of the Particle Accelerator Conference, Dallas, TX, 1995 (IEEE, New York, 1995), p. 2485.

[22] N. Kurita, D. Martin, S. Smith, C. Ng, M. Nordby, and C. Perkins, in Proceedings of the Particle Accelerator Conference, Dallas, TX, 1995 (IEEE, New York, 1995), p. 2512.

[23] John Corlett, Cho-K. Ng, and Stephen R. Smith, in Proceedings of the Particle Accelerator Conference, Vancouver, BC, Canada, 1997 (IEEE, New York, 1997), p. 2116.

[24] R. Lill, G. Decker, J. Hoyt, X. Sun, and J. Wang, in Proceedings of the 14th Beam Instrumentation Workshop, Santa Fe, NM (LANL, Los Alamos, 2010), p. 260. 